\title{
Geographic difference in advanced gastric cancer prevalence and chemotherapy treatment results: could it really be an independent prognostic factor?
}

\author{
Junichi Sakamoto $\cdot$ Koji Oba
}

Published online: 2 March 2012

(c) The International Gastric Cancer Association and The Japanese Gastric Cancer Association 2012

\begin{abstract}
Although quite a few reports of retrospective studies have been published, definitive evidence for the source of the disparities between East and West associated with gastric cancer chemotherapy, safety, and efficacy has not been shown to date. In order to shed light on these long-lasting discussions, Hsu et al. [1] have accumulated published information from 25 randomized clinical trials and investigated geographic differences in the safety and efficacy of systemic chemotherapy for advanced gastric carcinoma.

According to their meta-analysis and meta-regression analysis, patients in Asian trials have an $8.2 \%$ lower incidence of grade 3-4 neutropenia and a $2.1 \%$ lower incidence of grade 3-4 diarrhea than patients in non-Asian trials. It is true that most gastric cancer oncologists and investigators have been realizing that there might be several factors favoring Asian patients. In colon cancer clinical trials using a fluorinated pyrimidine as the key drug, Haller et al. [2] reported more grade 3-4 adverse events and discontinuation of the treatment in United States patients compared with nonUnited States patients. Focusing on East Asia and the United States, grade 3-4 gastrointestinal toxicity was significantly higher (hazard ratio 3.62, 95\% confidence interval $2.11-6.20, p<0.01)$ in the United States. This disparity in the tolerability profiles of fluorinated pyrimidines could be explained as arising from: (1) genetic polymorphisms
\end{abstract}

This editorial refers to the article doi:10.1007/s10120-011-0106-5.

J. Sakamoto $(\bowtie)$

Young Leaders' Program in Healthcare Administration,

Nagoya University Graduate School of Medicine, Nagoya, Japan

e-mail: sakamjun@med.nagoya-u.ac.jp

K. Oba

Translational Research and Clinical Trial Center,

Hokkaido University Hospital, Hokkaido, Japan of genes involved in fluorinated pyrimidine metabolism, (2) differences in dietary folate intake, and (3) cultural differences in patients' behavior in using an oral self-administered drug. Moreover, Ajani et al., according to the result of their phase I study of the oral fluorinated pyrimidine S-1, plus cisplatin, have postulated that the efficacy of the CYP26 enzyme is higher in whites than in Asians [3]. They presume that this difference causes the conversion of ftorafur to active fluorouracil (FU) to occur at a faster rate, achieving a higher AUC of FU in whites than in Asians. From these results, the S-1 dose was reduced from $80 \mathrm{mg} / \mathrm{m}^{2}$ in a Japanese study to $50 \mathrm{mg} / \mathrm{m}^{2}$ in a global trial. Also, in a genomic analysis of the UGT1A1 enzyme, which is responsible for the glucuronization and detoxification of SN-38, the active metabolite of irinotecan, the UGT1A $1 * 28$ polymorphism was detected to differ between Caucasians and Asians [4]. A Japanese study has also demonstrated that the UGT1A $1 * 6$ polymorphism, rather than the UGT1A $1 * 28$ polymorphism, is a potential predictor of toxicity caused by irinotecan in Japanese patients [5]. All these complicated findings and assumptions have now alerted clinicians on the need to establish optimal regimens of various chemotherapeutic agents for diverse cancer populations.

Another finding from Hsu et al. [5] is that the frequent use of second-line chemotherapy was commonly observed in Asian trials, and this was assumed to lead to a better overall survival in Asian patients, whereas progression-free survival (PFS) is better in Western trials. This difference could be explained simply by the fact that two- to threedrug combination regimens are common as first-line chemotherapy in Western trials. In such cases, first-line chemotherapy might be able to show a better response and better PFS than the mono- or two-drug combination therapies routinely used in Asia. After the failure of first-line chemotherapy that has exhausted all promising 
chemotherapeutic agents, no favorable candidate for second-line chemotherapy is available. The answer to the question of whether the combined use of chemotherapeutic agents is more effective than the sequential use of a single agent is being addressed in a randomized phase II study being performed in Japan (unpublished results of the Trial for Advanced Stomach Cancer (TASC) trial performed by the Epidemiological and Clinical Research Information Network (ECRIN) investigators), and the results will be reported.

Recent debates regarding differences in survival outcomes between East and West have largely focused on issues regarding surgical technique, especially in the field of adjuvant therapy. In addition, with the advent of universal coverage health insurance in Japan, Japanese patients are more likely to be diagnosed with a node-negative state that can be cured by surgical resection. The question of variability in diagnostic criteria for malignancy has also been raised, noting that gastric cancer is diagnosed early according to nuclear and structural criteria in Japan even when invasion is absent according to the Western viewpoint [6]. If all these assumptions (i.e., stage migration, difference in pathological diagnosis between East and West etc.) are confirmed, it is probable that stage-stratified gastric cancer survival would be decreased in Western countries compared with that in Asian countries. However, objections to the hypotheses of geographic differences have been raised in studies performed in Asian-American gastric cancer patients in the United States [7] and in Canada [8]. Results from the Los Angeles County Cancer Surveillance Program demonstrated that, in California, Asian-Americans showed a significantly better probability of survival compared with non-Asians (hazard ratio 0.76, 95\% confidence interval $0.72-0.82, p<0.001$ ), regardless of the presence of a similar extent of surgery, and a similar treating facility [7]. Of note, nearly one-fourth of Asians in California are uninsured and they are thus less likely to receive preventive or timely healthcare. This disparity in healthcare access could indicate that Asians in California would be more likely to have delays in diagnosis and to present with more advanced disease. Similar studies have been performed by the British Columbia Cancer Agency in Canada, where universal coverage health insurance provides comprehensive medical access to all residents, and treatment policies are fairly standardized in accordance with practical guidelines. A proportional hazards model adjusted for age, grade, location, extent, chemotherapy, and surgery, did not show statistically significant differences in outcome between Asians and non-Asians. However, Asian Canadian patients who underwent curative gastric cancer resection showed a significantly lower risk of mortality compared with non-Asians (hazard ratio $0.60,95 \%$ confidence interval $0.41-0.87, p<0.001$ ) [8]. As far as these results are concerned, we have to be a bit sceptical about the widely accepted opinion that Asian treatments for gastric cancer are superior to Western therapies.

Regarding the difference in subtypes of gastric cancer, gastric cancer in the cardia and esophagogastric junction adenocarcinoma were reported to be more common in Caucasians than in Asians. However, a study looking at the chronological changes in esophagogastric junction adenocarcinoma in the Japanese population has demonstrated that the incidence has increased from 2.3 to $10 \%$ (a factor of more than 4 times) during the past 40 years [9]; i.e., it has become comparable to the incidence in Caucasians [10]. One possible explanation could be the rapid expansion of programs for the eradication of Helicobacter pylori infection among Japanese. Since the 1990s, when H. pylori was determined as the major causative organism of atrophic gastritis, and the precancerous nature of atrophic gastritis for intestinal gastric cancer was shown, the eradication of $H$. pylori, using amoxicillin and levofloxacin hydrate, has been extensively performed in the older Japanese population at risk. As a result, the incidence of intestinal-type cancer has been decreased. However, in contrast, the preservation of gastric acid secretion due to the removal of $H$. pylori could have led to the development of esophagogastric junction diffuse-type adenocarcinoma in Japanese [11]. The prevalences of the different types and locations of gastric cancer may have been changing rapidly in certain Asian populations, especially among Japanese.

The most critical limitation of the study by Hsu et al. [11] is that the data obtained are mostly from the tabulated meta-analysis data of randomized clinical trials. It is now taken for granted that the meta-analysis of individual patient data will be able to give more detailed answers to most of the above-mentioned questions. Meta-analysis or meta-regression analysis may be biased to a certain extent by the lack of individual data for the patients enrolled in each clinical trial.

All in all, we should wait for the results of the Global Advanced/Adjuvant Stomach Tumor Research International Collaboration (GASTRIC) investigators' individual patient data meta-analysis [12] before further proclaiming the importance of geographic differences in the safety and efficacy of chemotherapies for gastric or esophagogastric junction adenocarcinoma.

Acknowledgments We thank the ECRIN investigators for providing unpublished findings from their TASC clinical trial.

\section{References}

1. Hsu C, Shen YC, Cheng CC, Cheng AL, Hu FC, Yeh KH. Geographic difference in safety and efficacy of systemic chemotherapy for advanced gastric or gastroesophageal carcinoma: a meta-analysis and meta-regression. Gastric Cancer. 2011 (in press). 
2. Haller DG, Cassidy J, Clarke SJ, Cunningham D, Van Cutsem E, Hoff PM, et al. Potential regional differences for the tolerability profiles of fluoropyrimidines. J Clin Oncol. 2008;26(13): 2118-23.

3. Ajani JA, Faust J, Ikeda K, Yao JC, Anbe H, Carr KL, et al. Phase I pharmacokinetic study of $\mathrm{S}-1$ plus cisplatin in patients with advanced gastric carcinoma. J Clin Oncol. 2005;23(28):6957-65.

4. Liu JY, Qu K, Sferruzza AD, Bender RA. Distribution of the UGT1A $1 * 28$ polymorphism in Caucasian and Asian populations in the US: a genomic analysis of 138 healthy individuals. Anticancer Drugs. 2007;18(6):693-6.

5. Takano M, Kato M, Yoshikawa T, Goto T, Furuya K, Kikuchi Y. Indispensability of UGT1A1*6 genotyping in Japanese cancer patients treated with irinotecan. Int J Clin Oncol. 2010;15(2): 224-5.

6. Schlemper RJ, Itabashi M, Kato Y, Lewin KJ, Riddell RH, Shimoda $\mathrm{T}$, et al. Differences in diagnostic criteria for gastric carcinoma between Japanese and western pathologists. Lancet. 1997;349(9067):1725-9.

7. Kim J, Sun CL, Mailey B, Prendergast C, Artinyan A, Bhatia S, et al. Race and ethnicity correlate with survival in patients with gastric adenocarcinoma. Ann Oncol. 2010;21(1):152-60.
8. Gill S, Shah A, Le N, Cook EF, Yoshida EM. Asian ethnicityrelated differences in gastric cancer presentation and outcome among patients treated at a Canadian cancer center. J Clin Oncol. 2003;21(11):2070-6.

9. Kusano C, Gotoda T, Khor CJ, Katai H, Kato H, Taniguchi H, et al. Changing trends in the population of adenocarcinoma of the esophagogastric junction in a large tertiary referral center in Japan. J Gastroenterol Hepatol. 2008;23(11):1662-5.

10. Kubo A, Corley DA. Marked multi-ethnic variation of esophageal and gastric cardia carcinomas within the United States. Am J Gastroenterol. 2004;99(4):582-8.

11. Koike T, Ohara S, Inomata Y, Abe Y, Iijima K, Shimosegawa T. The prevalence of Helicobacter pylori infection and the status of gastric acid secretion in patients with gastroesophageal junction adenocarcinoma in Japan. Inflammopharmacology. 2007;15(2): $61-4$.

12. GASTRIC Group, Paoletti X, Oba K, Burzykowski T, Michiels S, Ohashi Y, Pignon JP, et al. Benefit of adjuvant chemotherapy for resectable gastric cancer: a meta-analysis. JAMA. 2010;303(17): 1729-37. 